\title{
Discharge flow of a granular media from a silo: effect of the packing fraction and of the hopper angle
}

\author{
Mebirika Benyamine ${ }^{1}$, Pascale Aussillous ${ }^{2, \star}$, and Blanche Dalloz-Dubrujeaud ${ }^{2}$ \\ ${ }^{1}$ Laboratoire FIMAS, Université de Béchar, B.P 417 Béchar 08000, Algérie \\ ${ }^{2}$ Aix-Marseille Univ., CNRS, IUSTI UMR 7343, 13013 Marseille, France
}

\begin{abstract}
Silos are widely used in the industry. While empirical predictions of the flow rate, based on scaling laws, have existed for more than a century (Hagen 1852, translated in [1] - Beverloo et al. [2]), recent advances have be made on the understanding of the control parameters of the flow. In particular, using continuous modeling together with a mu(I) granular rheology seem to be successful in predicting the flow rate for large numbers of beads at the aperture (Staron et al.[3], [4]). Moreover Janda et al.[5] have shown that the packing fraction at the outlet plays an important role when the number of beads at the apeture decreases.

Based on these considerations, we have studied experimentally the discharge flow of a granular media from a rectangular silo. We have varied two main parameters: the angle of the hopper, and the bulk packing fraction of the granular material by using bidisperse mixtures. We propose a simple physical model to describe the effect of these parameters, considering a continuous granular media with a dilatancy law at the outlet. This model predicts well the dependance of the flow rate on the hopper angle as well as the dependance of the flow rate on the fine mass fraction of a bidisperse mixture.
\end{abstract}

\section{Introduction}

The prediction of the mass flow rate of a granular media discharged from a silo has been widely studied because of its practical interest in several industries, for example to ensure reproducible and efficient handling in plant operations (food, mining, ceramic, paint, pharmaceutical... industries). One of the simplest and widely used expression is the one proposed by Berverloo [2] for flat-bottomed silos. Following the experimental observation that the flow rate is mainly controlled by the outlet diameter $D$, it is based on dimensional analysis with a concept of a free fall arch at the outlet [1] and of an "empty annulus" [6] considering a reduced outlet length due to the particle size. More recent works proposed a continuous modeling with a mu(I) granular rheology which gives good results for a large amount of beads at the aperture [3], [4]. However, the control parameters of the flow are not well understood. In this paper we investigate the influence of the discharge angle of the hopper, and the bulk packing fraction of the granular material (using bidisperse mixtures). Our results are interpreted through a physical and analytical model (based on the work of Janda et al.[5]) considering a modification of the density close to the outlet due to the flow.

\section{Experimental methods}

In order to measure the flow rate and to observe the granular flow, the experimental setup is made of a rectangular

\footnotetext{
^e-mail: pascale.aussillous@univ-amu.fr
}

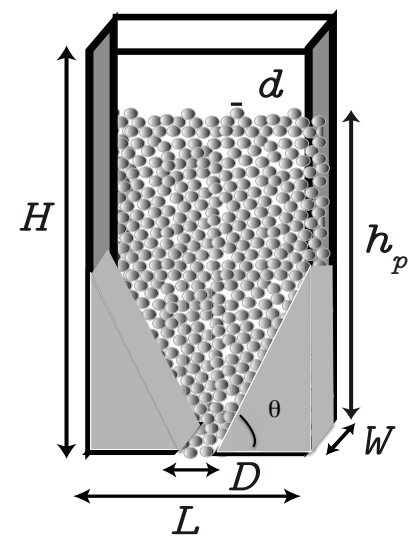

Figure 1. Experimental setup: rectangular silo with adjustable outlet $D$ and discharge angle $\theta$.

silo (height $H=1 \mathrm{~m}$, width $L=60 \mathrm{~mm}$, and thickness $W$ which can be varied from 3.5 to $10 \mathrm{~mm}$ ) made with two parallel plates (metal/PMMA for the flat bottom experiments and glass/glass for the study with several discharge angles) (Fig. 1). The rectangular outlet located at the centre of the bottom spans the thickness $W$ and its length $D$ can be varied from 1.8 to $26 \mathrm{~mm}$. The discharge angle $\theta$ of the outlet to the horizontal can also be varied from $\theta=0^{\circ}$ to $\theta=80^{\circ}$. When opening manually the outlet, the grains fall out of the silo and are collected in a vessel. The temporal evolution of the mass $m(t)$ is recorded using 


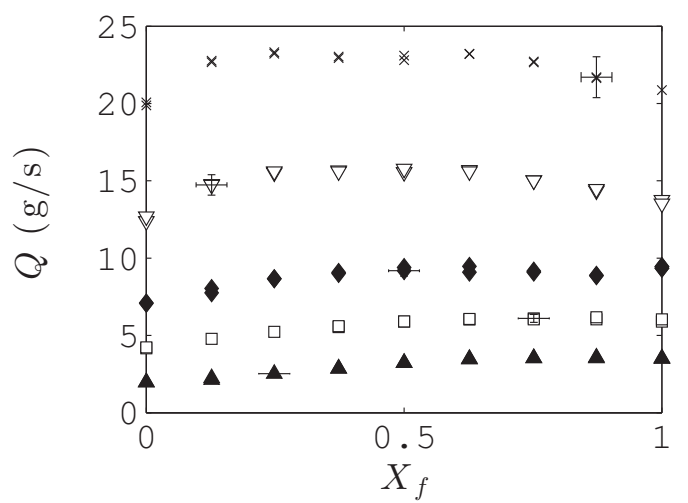

Figure 2. Flow rate versus $X_{f}$ for binary mixtures $(r=6.3$,

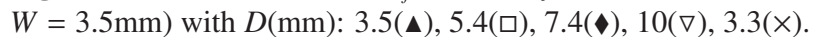

an electronic scale. A high speed camera allowed us to visualise the flow at the wall. All the experiments have been carried out in the stationary flow regime.

The granular material consists of smooth spherical glass beads (density $\rho=2500 \mathrm{~kg} \cdot \mathrm{m}^{-3}$ ) with different diameters $d$ from 80 to $4500 \mu m$ (with a dispersion of $\pm 10 \%$ ). With the particles and aperture available, the number of beads across the aperture $(D / d)$ ranges between 1 and 325 (clogging occurs for $D / d<4.1$ [7]). In order to control the bulk volume fraction, different binary mixtures were tested (size ratio of coarse particles diameter on fine particules diameter $r=d_{c} / d_{f}$ between 2.2 to 6.3). Controlling the mass fraction $X_{f}$ of the fine particles, the bulk volume fraction varies from 0.58 to 0.73 . To ensure the homogeneity of the bi-disperse mixture in the silo, ten layers of the desired fine mass fraction $\left(X_{f}\right)$, previously mixed by hand, were poured successively and carefully into the silo. The packing fraction $\phi_{b}$ for each experiment has been calculated measuring the height and the weight of the grain assembly in the silo. Mono-disperse and bi-disperse experiments have been carried out, for the bi-disperse mixtures, all the experiments have been performed with an angle $\theta=0^{\circ}$.

\section{Results and discussion}

In our configuration the Beverloo law becomes:

$$
Q=C \rho \phi_{b} g^{1 / 2} W(D-k d)^{3 / 2}
$$

Where $\phi_{b}$ is the initial bulk particle volume fraction and C and $\mathrm{k}$ are fitting parameters. This expression fits well all our experimental results for mono-disperse experiments, but it is difficult to modify this expression in the case of a binary mixture even if $\phi_{b}$ is known. Indeed, for a bidisperse mixture, we observe that the flow rate always exceeds the rate predicted by a simple mixture law between the flow rate of the purely coarse particles and the purely fine particles (Fig. 2). A mixture law to express the mean diameter of the particules is not suitable to predict the flow rate.

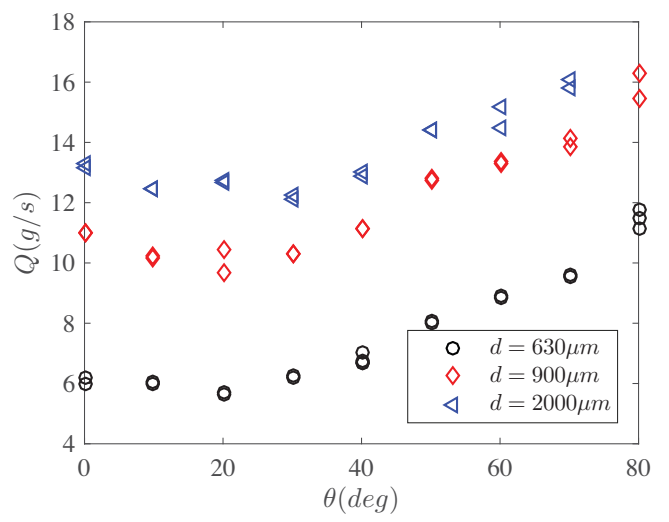

Figure 3. Flow rate versus the discharge angle $\theta$ for $D=8 \mathrm{~mm}$

We also measure the flow rate when the discharge angle is increased from $\theta=0^{\circ}$ to $\theta=80^{\circ}$. In agreement with the literature [8],[9], the flow rate increases significantly for a discharge angle $\theta>20^{\circ}$ as shown in Fig 3 .

Thus, to interpret our results, we have adapted the model proposed by Janda et al. [5]. This model is based on the experimental measurement of the velocity and the density profile at the outlet. The author found that both these profiles are self similar. Thus the flow rate is given by the velocity $v_{o}$ and the density $\phi_{0}$ at the outlet, $Q=$ $\rho \iint_{S_{o}} \phi(x) v(x) d x=C \rho W D \phi_{0} v_{0}$. Measuring this quantity these authors have shown that for a given outlet diameter, the particle velocities are the same whatever the particle diameter $\left(v_{0} \approx \sqrt{g D}\right)$. Moreover, they have found that, instead of considering a reduce outlet, a dilatancy at the outlet should be taken into account $\left(\phi_{0} \approx \phi_{b}\left[1-\alpha_{1} e^{-\alpha_{2} D}\right]\right)$. A recent work [10] has shown that close to the outlet, the particles are accelerated leading to the scaling of the velocity, but not in an idealised free fall. We adapted this model to propose a new expression for the flow rate :

$$
Q=C \rho \phi_{0} \sqrt{g D^{3}} W=C \rho \phi_{b} G(D / d) \sqrt{g D^{3}} W
$$

with the dilatancy function $\left(\phi_{0} / \phi_{b}\right)$,

$$
G(D / d)=\left[1-\alpha e^{-\beta \frac{D}{d}}\right]
$$

Equation 2 fits well our experimental results as shown on Fig 4 and allowed us to calculate the parameters $C=0.9$, $\alpha=0.66$ and $\beta=0.11$.

This is then possible to calculate the experimental dilatancy function given by :

$$
G(D / d)=\frac{\phi_{o}}{\phi_{b}}=\frac{Q}{C \rho \phi_{b} \sqrt{g D^{3}} W}
$$

The evolution of the dilatancy function $G(D / d)$ with $D / d$ (which represents the number of bead at the aperture) is given in fig. 5 for a mono-disperse flow in a flat bottom silo and is well fitted by equation 3. This result was also obtained numerically using discrete simulations by Zhou et al. [11]. 


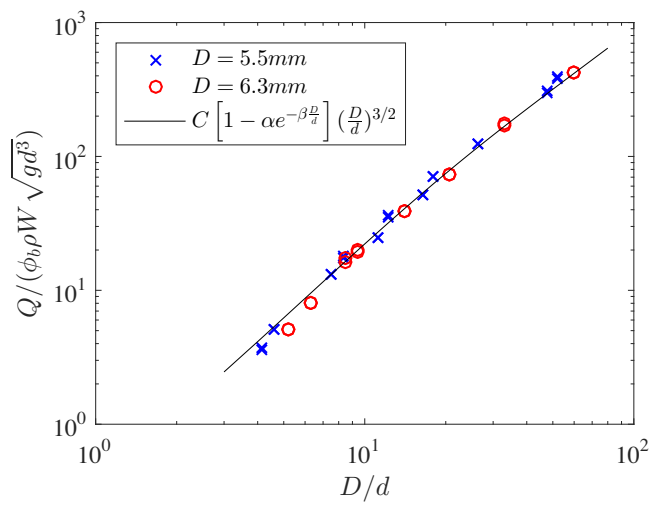

Figure 4. Dimensionless flow rate versus $D / d$ for two outlets size D. The black line represents equation 2 with $C=0.9, \alpha=$ 0.66 and $\beta=0.11$

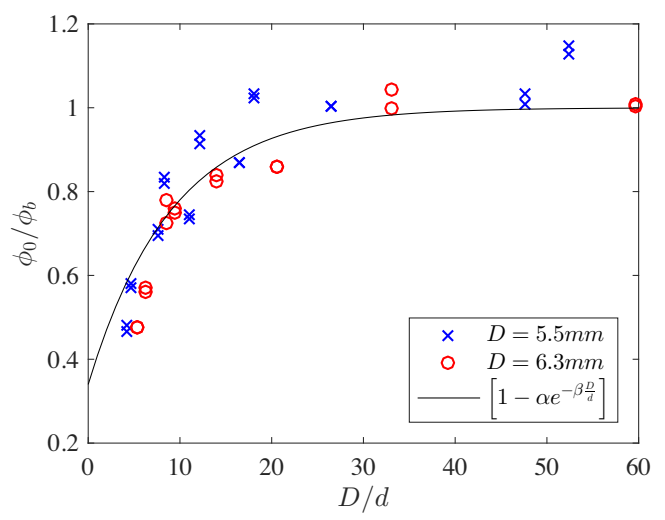

Figure 5. Dilatancy at the outlet for $W=3.5 \mathrm{~mm}$. Theoretical curve (equation 3) obtained with $\alpha=0.66$ and $\beta=0.11$

\subsection{Bidisperse mixtures}

We assume that for a bidipserse mixture, the same hypothesis are still and independently valid for each size of particles $d_{i}$. Each population of particles has his own dilatancy function $G(d i / D)$. The dilatancy at the outlet is then simply given by $\phi_{0}=\phi_{0 f}+\phi_{0 c}$, and the flow rate for a bi-disperse mixture becomes :

$$
Q=C\left[X_{f} G\left(\frac{D}{d_{f}}\right)+\left(1-X_{f}\right) G\left(\frac{D}{d_{c}}\right)\right] \rho \phi_{b} W \sqrt{g D^{3}}
$$

Equation 5 suggests that the dilatancy at the outlet is given by a simple mixture law between the dilatancy factor of the fine and the coarse particles. Note that $\phi_{b}$ depends also on the fine mass fraction, which implies a complex variation of the flow rate with $X_{f}$ as seen in Fig. 2. This model fits well our experimental results represented in Fig. 6 , where the dilatancy at the outlet is plotted versus the fine mass fraction for three mixtures. More details can be found in [7].

Our model suggests that a mixture diameter cannot be simply defined to adapt the monodisperse expressions to predict the bidisperseflow rate as commonly seen in the literature [12], [13].

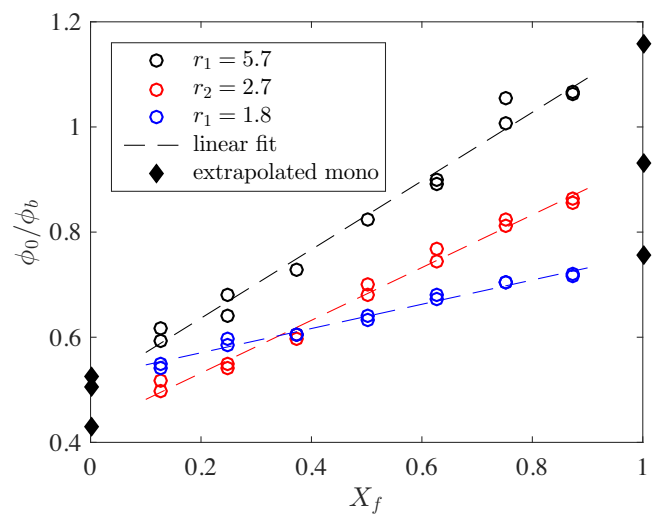

Figure 6. Linear evolution of the dilatancy parameter with $X_{f}$ for bidisperse Mixture. Monodisperse results have been excluded from the linear fit, and represent the extrapolated point for monodisperse flow.

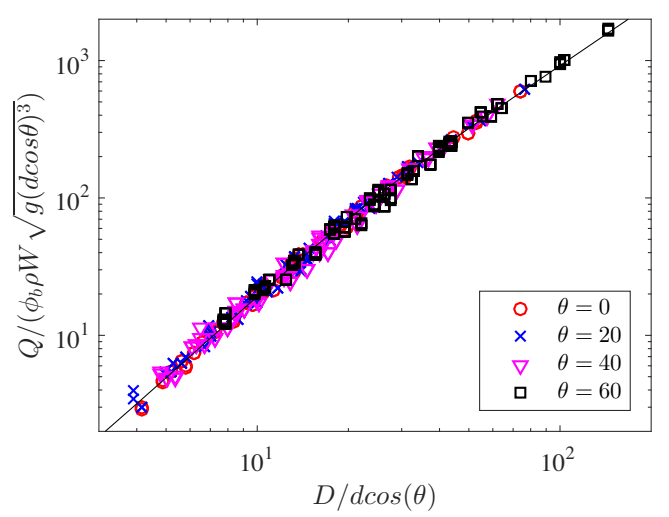

Figure 7. Dimensionless flow rate versus $D /(d \cos \theta)$ for several discharge angles. The black line represents equation 7 with $C=$ $0.92, \alpha=0.80$ and $\beta=0.085$.

\subsection{Discharge angle}

In this part, all the presented results have been obtained with monodisperse material, varying the discharge angle and presented on Fig. 3. We made the assumption that the self similarity hypotheses are still valid for the velocity and the density profile, and that the modification of the flow rate is only due to the dilatancy factor which must depend on the discharge angle. Indeed, to allow the granular media flowing through the outlet, a singular region exist above the outlet, where the particle are accelerated. We suppose that this region is delimitated by an arch of radius of curvature $\mathcal{R}$, corresponding to the convergence of the velocity stream through the outlet. For a discharge angle of $\theta=90^{\circ}$ (i.e. a vertical tube), the velocity stream are parallel and this radius of curvature is infinite. We suppose that the the evolution of $\mathcal{R}$ is continuous with the discharge angle and the simplest function we can suggest is $\mathcal{R}=(D / 2) \cos (\theta)$, which recovers the typical length scale $D / 2$ for the flat bottom. Thus, a new expression is proposed for the dilatancy function:

$$
G(D / d, \theta)=\left[1-\alpha e^{-\beta \frac{D}{d \cos \theta}}\right]
$$




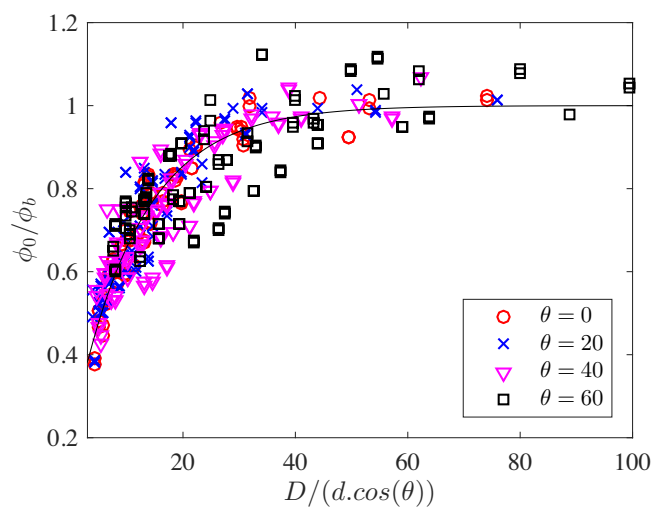

Figure 8. dilatancy at the outlet versus $D /(d \cos \theta)$ for several discharge angles. The black line represents equation 6 with $\alpha=$ 0.80 and $\beta=0.085$.

Using this function, we obtain for the flow rate

$$
Q=C G\left(\frac{D}{d \cos \theta}\right) \rho \phi_{b} W \sqrt{g D^{3}}
$$

In figures 7 and 8 we have plotted the dimensionless flow rate and the dilatancy function, for several angles of discharge, as a function of $D /(d \cos \theta)$. In this representation the data collapse fairly well and are well fitted by equations 6 and 7 with the parameter $C=0.92, \alpha=0.80$ and $\beta=0.085$ obtained in our apparatus using the flat bottom data.

\section{Conclusion}

Using experiments we have proposed a model which predicts the flow rate of a granular media discharging from silos taking into account the discharge angle of the hopper and the bulk packing fraction of the granular material through a dilatation factor. The perspective of this work is to validate the hypothesis proposed thanks to velocity and density profiles measurement close to the outlet.

\section{Acknowledgements}

This work has been carried out in the framework of the Labex MEC (ANR-10-LABX-0092) and of the A*MIDEX project (ANR-11-IDEX-0001-02), funded by the "Investissements d'Avenir" French Government program managed by the French National Research Agency (ANR).

\section{References}

[1] B. Tighe and M. Sperl, Granul. Matter 9, 141 (2007)

[2] W. A. Beverloo, H. A. Leniger, and J. V. de Velde, Chem. Eng. Sci. 15, 260 (1961)

[3] L. Staron, P.-Y. Lagrée, and S. Popinet, Phys. of Fluids, 24,103301 (2012)

[4] L. Staron, P.-Y. Lagrée, and S. Popinet, Eur. Phys. J. E 37,5 (2014)

[5] A. Janda, I. Zuriguel, and D. Maza, PRL 108, 248001 (2012)

[6] R. L. Brown and J. C. Richards, Trans. Inst. Chem. Engrs. 38, 243 (1960)

[7] M. Benyamine and M. Djermane, B. DallozDubrujeaud and P. Aussillous, PRE 90, 032201 (2014)

[8] R. M. Nedderman, Cambridge University Press, (1992).

[9] H. F. Rose and T. Tanaka, The Engineer (London) 208 Oct. 23 (1959)

[10] S.M. Rubio-Largo, A. Janda, D. Maza, I. Zuriguel, and R. C. Hidalgo, PRL 114, 238002 (2015)

[11] Y. Zhou, P. Ruyer, and P. Aussillous, PRE 92, 062204 (2015)

[12] S. Humby, U. Tüzün, and A. B. Yu, Chem. Eng. Sci. 53, 483 (1998).

[13] P. Arteaga and U. Tüzün, Chem. Eng. Sci. 45,205(1990) 